Review Article

doi:10.29052/IJEHSR.v6.i1.2018.47-57

\title{
Oral flora: protection or destruction of dental tissue
} Merina Shakya ${ }^{1 \pm}$, Pujan Kranti Kayastha ${ }^{2 \pm}$ \& Huang Jiao $^{1 *}$

${ }^{1 .}$ Department of Periodontics, Stomatological Hospital of Chongqing Medical University, Chongqing Medical University, Chongqing, China.

2. Department of Conservative Dentistry and Endodontics, Stomatological Hospital of Chongqing Medical University, Chongqing Medical University, Chongqing, China.

Corresponding Author Email ID: 3547747852@qq.com

Received 24/2/18; Accepted 30/3/18; First Published 31/3/18

\pm both author contributed equally and therefore considered as first joint co-author

\section{Abstract}

Background: Oral cavity contains heterogenous environment which provides different niches in different environment present in a symbiotic relation. Normal oral flora when getting favorable environment gets attached to the tooth surface. Alteration in this mutualistic association transforms into diseased condition. The oral cavity begins to harbor microflora immediately after birth. Oral cavity harbors about 20 phyla and more than 700 species. The objective of this study is to determine the origin of the microorganisms responsible for dental diseases.

Methodology: PUBMED database was searched for the English articles published with the combinations of following search terms: normal oral bacteria, oral bacteria, oral microbiome, dental caries, tooth caries, endodontic infection, and recurrent pulpal infection. Abstracts and also full text was revised to identify the suitable papers that describe the microbiological association of dental diseases which were used.

Results: Nosocomial infection has found to be associated with persistent endodontic infection. Thus, proper sterilization and change of instruments and gloves after each procedure for every root canal instrumentation are mandatory to provide clean sterile preparation of the canal. Also, persistent/secondary intraradicular infection associated with Actinomyces species and P. propionicum is treated with surgical procedure only. Therefore bacteriological analysis of the canal helps to determine the efficacy of the endodontic treatment in primary infection, chances of secondary or persistent endodontic infection, and requirement of surgical treatment.

Conclusion: Dental diseases are mainly associated with the virulence of different microorganisms present in the oral cavity. After the initiation of disease, exogenous bacteria are attracted to the site. Thus maintenance of proper oral hygiene prevents the transformation of normal flora to a diseased state. If the disease is diagnosed early and treated, it prevents the life-threatening condition.

\section{Keywords}

Dental Caries, Endodontic Infection, Normal Oral Flora, Oral Microbiome, Recurrent Infection

\section{Introduction}

The human body is colonized with different microbes present in the mucosal surface of the oral cavity along with gastrointestinal tract, urogenital tract, and the surface of the skin. Those microbes show permanent colonization in a symbiotic relationship producing beneficial results. Alteration in the symbiotic relation of oral microbes leads to transformation of opportunistic pathogens and causes diseases. The oral cavity being unique, contain diverse microflora distributed in various niches and harbors more than 700 species of microorganisms ${ }^{1}$. The oral cavity contains 20 phyla with the majority of sequences belonged to one of the seven phyla: Actinobacteria, Bacteroides, Firmicutes, Fusobacteria, Proteobacteria, Spirochetes, and candidate division TM7, depending on age/dentition stage. ${ }^{2}$ The oral cavity 
begins to harbour microflora immediately after birth through continuous contacts with microbes outside the sterile intra-uterine and continues through the remainder of life. Multiple factors like delivery mode, feeding habit, eruption and shedding of tooth promote differentiation of bacterial communities ${ }^{2,3}$. The oral cavity contains different surfaces like teeth, gingival sulcus, attached gingiva, tongue, cheek, lip, hard and soft palate which provides several distinct habitats for microbial colonization. These habitats in oral cavity provide a suitable environment for significantly different microbial communities and therefore bacteria predominant in one specific site differ from other site ${ }^{2,4}$. Destruction of protective layer primarily initiates the formation of dental caries.

\section{Dental Caries}

Dental caries is one of the world's most prevalent diseases. It is a complex interaction between the commensal microbiota, host susceptibility and environmental factors, such as diet, time and acid produced by bacteria that degrade tooth structure, leading to demineralization and cavitation. This complex biofilm mainly contains acidogenic and acidophilic bacteria which is responsible for acid production that decreases the $\mathrm{pH}$ and provides the acidic environment. Acidic environment favors the tooth enamel barrier breakdown leading to carious lesion formation extending into underlying hard tissue $^{5,6}$. Dental caries is due to endogenous bacteria that shift from normal mutualistic state to diseased state $^{7}$. As caries progresses, change in the composition of microbiota in the oral cavity is observed.

Streptococcus mutans acidifies the biofilm that results in attraction of acidogenic-aciduric bacterial species along with Actinomyces and Lactobacilli that are recognized to be involved in cariogenic processes including early childhood caries, white spot lesions, cavitated lesions, or carious dentin ${ }^{8}$. S.mutans's virulence factor is able to convert dietary sucrose into a diverse range of soluble and particularly insoluble extracellular polysaccharides through exoenzymes such as glucosyltransferases and fructosyltransferase. These extracellular polysaccharides are the prime building blocks of cariogenic biofilms that promote colonization of S. mutans and recruitment of additional microorganisms into dental plaque. It is found that the combination of $\mathrm{S}$. mutans with $\mathrm{C}$. albicans enhance acid production, due to the high acidogenic property of the fungus ${ }^{9}$.

Compared to enamel, dentine carious lesion provides a completely different scenario for residing microbes. Bifidobacterium and S.mutans may be involved in initiation and progression of dental caries. Deep into the dentin, S. mutans might lose its dominant role being outcompeted by obligate anaerobes like Propionibacteria which enables to degrade proteins from exposed dentinal collagen network. Propionibacterium acidifaciens is reported to be saccharolytic, producing large amounts of acetic and propionic acids and are increasingly reported to be present in deep dentin and root caries ${ }^{6}$. Presence of Lactobacilli in the sample causes a decrease in a number of Prevotella spp. and no Pseudoramibacter and vice versa. According to Chhour et al. (2005), Lactobacillaceae and Prevotellaceae make the majority of all identified sequences. Depending on the metabolic by-products, dominant species could be grouped into Prevotella-dominated or Lactobacillusdominated samples. The dominant species in the carious lesions depends on the metabolic byproducts of the initial colonizers ${ }^{5}$.

Carious tooth contains various $\mathrm{pH}$ within it due to which microbial communities in low $\mathrm{pH}$ zone differ from that of high $\mathrm{pH}$ zone. Most superficial zone was found to be significantly more acidic than the deepest areas of the sampled lesion. As the $\mathrm{pH}$ increases, Firmicutes decreases but other phyla such as: Bacteroidetes, Fusobacteria and Proteobacteria becomes dominant (increases). Kianoush (2014) found that L. fermentum, L. rhamnosus, and L. crispatus were predominant in acidic $\mathrm{pH}$ and Sphingomonas sp, S. oralis, Lachnospiraceae sp., Atopobium rimae and Bifidobacterium dentium in basic $\mathrm{pH}$. The microbiota which appeared to be 
unaffected by $\mathrm{pH}$ includes Leptotrichia spp., Prevotella spp., Streptococcus salivarius and candidate division $\mathrm{TM} 7^{10}$. Alloprevotella tanerrae, Leptothrix sp., Sphingomonas sp. and Streptococcus anginosus were predominant in neutral $\mathrm{pH}$. Types and numbers of microorganisms in the dentinal carious lesion is greatly influenced by the environment. The dominant phyla found in the dentinal carious lesions are Firmicutes, Actinobacteria, and Bacteroidetes. Most predominant genera in the oral cavity are Lactobacillus, Atopobium, Prevotella, Olsenella, Actinomyces, Streptococcus, Propionibacterium, Bifidobacterium, Dialister, Sphingomonas, Fusobacterium, Parascardovia, Selenomonas, Scardovia, Chryseobacterium, Terrimonas, Burkholderia and Sporobacter ${ }^{10,11}$.

Bacterial species present in dentinal caries varies to the location as coronal and radicular. The most abundant species present in root caries are Lactobacillus gasseri, Prevotella denticola, Alloprevotella tannerae, S. mutans and Streptococcus sp.HOT $070^{10}$. Ma (2015) found Actinomyces spp., S. mutans, S. sobrinus, Lactobacilli, V. parvula, R. dentocariosa, P. micra, $P$. acnes and N. mucosa are predominant in initial carious root lesions ${ }^{12}$. Zaremba (2006) found Peptostreptococcus spp., Staphylococcus spp, Streptococcus spp, Actinomyces spp, Nisseria spp, Veionella spp, and Candida spp are present in root surface caries. Candida spp. were also isolated from root caries lesions of adult subjects ${ }^{13}$. The Streptococcus and Veillonella genera produce lactate, and have been associated with Early Childhood Caries $^{14}$. S. mutans, S. sobrinus, Bifidobacteriaceae, Scardovia wiggsiae, Porphyromonas catoniae, Actinomyces, R.mucilaginosa, Prevotella species and Slackia exigua ${ }^{8,12,15}$, and the combinations of S. mutans with S. sobrinus, S. mutans with Bifidobacteriaceae and S. mutans with Scardovia wiggsiae were also found to be associated with Severe Early Childhood Caries $^{12,15}$. If dental caries left untreated, bacteria from carious lesions progress deep into dental pulp that leads to Pulpal infection which may further progress to space infection.

\section{Endodontic infection}

Infection of the dental Pulpal tissues is caused by the necrosis of dental pulp or dental root canal. It is the leading cause of oro-facial pain, localized and spreading dental infections and loss of teeth ${ }^{16}$. Along with dental caries, the infection gets entry to dental pulp via dentinal tubules, direct pulp exposure, periodontal disease and anachoresis. Whenever the distance between pulp and remaining healthy dentine is less than $0.2 \mathrm{~mm}$, bacteria gain access to the pulp through dentinal tubules in a centripetal direction. Microorganism might infect healthy tooth to infected tooth via lateral canal or apical foramen or may get an entry from periodontal membrane through the accessory canal or apical foramen.

Although more than 700 types of microbial species have been detected from the infected root canal, only $40 \%$ have been identified. The number of bacteria per canal per tooth varies from sample to sample. The number of different bacterial species

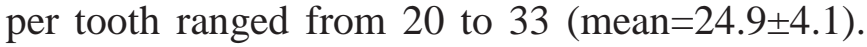
The number of different bacterial species detected per root canal ranged from 5 to 33 $(\text { mean }=20.0 \pm 7.9)^{17}$. Differences in endodontic bacteria are most likely to be a result of the differences in the composition of the oral microbiota. Studies have shown that number of bacterial species and cells in the root canal is dependent on the size of the periapical lesion. Larger the size of the lesions more bacterial species and longer the time period of the infection the more complex association of bacteria are present ${ }^{18}$.

The etiology of endodontic infections is heterogeneous and is likely to be polymicrobial ${ }^{16}$. Bacterial profiles of endodontic microbiota vary from individual to individual i.e., each individual harbors a unique endodontic microbiota in terms of species, richness, and abundance. (See table 1) Anaerobic microorganisms were found in $95 \%$ of the samples, black-pigmented bacilli in $37.5 \%$, 
aerobic microorganisms in $92.5 \%$, streptococci in $95 \%$, and Streptococcus mutans in $45 \%{ }^{19}$ while in another experiment it has isolated $83 \%$ facultative species, $100 \%$ anaerobic species, $75 \%$ aerobic species and $96 \%$ Black-pigmented bacilli ${ }^{17}$. Endodontic bacteria fall into 8 of the 13 phyla that have oral representatives, namely Firmicutes, Bacteroidetes, Spirochetes, Fusobacteria, Actinobacteria, Proteobacteria, Synergistes, and TM7. Al-Samahi (2014) isolated only 4 out of seven common phyla: Firmicutes, Bacteroidetes, Actinobacteria, Proteobacteria ${ }^{20}$ whereas Santos (2011) have found that Firmicutes, Bacteroidetes, Fusobacteria, Actinobacteria and Proteobacteria, collectively constituted more than $90 \%$ of the microbiome ${ }^{21}$. The most frequent bacterial species found in one study were Fusobacterium nucleatum sp.nucleatum, Fusobacterium periodonticum, Prevotella melaninogenica, Prevotella nigrescens, Prevotella intermedia, Enterococcus faecalis, Gemella morbillorum and Parvimonas micra 17 whereas Campylobacter gracilis, Eubacterium tardum, Peptostreptococcus anaerobius, Peptostreptococcus micros and members of the Lachnospiraceae were detected in another study. ${ }^{19}$ Fusobacteria were predominant in acute than in chronic cases whereas Eubacterium and Mogibacterium were the most prevalent in chronic cases. Bacteroidetes and Actinobacteria were present in acute and chronic cases ${ }^{21,22}$.

Table 1: Bacteria isolated from primary endodontic infection

Strict anaerobic bacteria gram-negative rods

\begin{tabular}{ll}
\hline Porphyromonas & P. gingivalis, P. endodontalis, \\
\hline Prevotella & $\begin{array}{l}\text { P.denticola, P.intermedia, P. nigrescens, P. tannerae, } \\
\text { melaninogenica,P. buccae, P. buccalis, P. oralis, P. loescheii }\end{array}$ \\
\hline Fusobacterium & F. nucleatum, F. periodonticum \\
\hline Treponema & $\begin{array}{l}\text { T. denticola, T. vincentii, T. socranski, T. parvum, T. maltoph } \\
\text { T.lecithinolyticum }\end{array}$ \\
\hline Tannerella & T. forsythia \\
\hline Obligately anaerobic gram negative coccobacilli \\
\hline Dialister & D. invisus, D. pneumosintes \\
\hline Strict anaerobic Gram-positive rods \\
\hline Propionibacterium & P. acnes, P. propionicum, \\
\hline Pseudoramibacter & Pseudoramibacter alactolyticus \\
\hline Olsenella & O. uli \\
\hline Filifactor & Filifactor alocis \\
\hline Eubacterium & E. alactolyticum, E.lentum, E. timidum, E. brachy, E. nodatum \\
\hline Strict anaerobic Gram-positive cocci \\
\hline Peptostreptococcus & P. anaerobius, P. micros (now named as Parvimonas micra) \\
\hline Gram negative cocci & C.gingivalis, C. ochracea, C. sputigena \\
\hline Capnocytophaga & C. rectus, C. curvus, C.gracilis \\
\hline Campylobacter & V. parvula \\
\hline Veillonella & E faecalis, E. faecium, E. hirae \\
\hline Facultative anaerobic bacteria Gram-positive cocci \\
\hline Enterococcus & S.anginosus, S. sanguis, S. mitis, S. oralis, S.gordonii \\
\hline Streptococcus & S. haemolyticus, \\
\hline Staphylococcus & \\
\hline Facultave anaer &
\end{tabular}

Facultative anaerobic gram positive rods

\begin{tabular}{ll}
\hline Actinomyces & A. israelii, A. odontolyticus, \\
\hline Lactobacilli & L. gasseri, \\
\hline
\end{tabular}




\begin{tabular}{l}
\hline Uncultivated phylotypes to genera \\
\hline Synergistes \\
\hline Dialister \\
\hline Prevotella \\
\hline Solobacterium \\
\hline Eubacterium \\
\hline Megasphaera \\
\hline Lachnospiraceae
\end{tabular}

Teeth with intact crowns but with necrotic pulps in root canal harbors strict anaerobes, usually belonging to genera Fusobacterium, Porphyromonas, Prevotella, Eubacterium, and Peptostreptococcus. When the direct communication is present with oral cavity, root canal harbors facultative anaerobic and aerobic bacteria. Root canals which remain open during the treatment harbors enteric bacteria more frequently and are more resistant to endodontic treatment ${ }^{23}$. Most studies revealed the higher occurrence of gram-positive bacteria (e.g., Streptococci, Lactobacilli, Enterococcus faecalis, O. uli, M. micros, P. alactolyticus, and Propionibacterium species) in both post-instrumentation and postmedication samples. As the infection progresses from coronal to apical part of the root canal which remains for a longer period of time leads to the condition in which facultative gram-positive bacteria is changed to gram-negative bacteria, due to change in nutritional supply and oxygen tension ${ }^{24}$. The coronal parts of the exposed root canal have exogenous nutrients (carbohydrates) and the body of the root canal has endogenous nutrients (proteins, glycoproteins). This variation influences the microbial ecology which leads to slow growing obligate anaerobes in apical site ${ }^{25}$. Proteins and glycoproteins also help to rise in the $\mathrm{pH}$ of the root canal and alteration in redox potential ${ }^{26}$. Metabolism of one species also provides the nutritional supply for the other synergistic bacteria. This shows the mutualistic relation among the bacteria present in the root canal ${ }^{25,27}$. Numerous studies have found the positive and negative association between the bacterial species in the canal. A positive association was found between Fusobacterium nucleatum and P. micros, P. endodontalis, C.rectus and Selenomonas sputigena, P.intermedia and P. micros, P.anaerobius and Eubacterium species. Eubacteria was associated with Peptostreptococcus while P. endodontalis was associated with Fusobacterium nucleatum, Eubacterium Alactolyticum and C. rectus and negative correlation between P. endodontalis and $\mathrm{P}$. intermedia. Propionibacterim propionicum, Capnocytophaga ochracea and veillonella parvula and other species were found to be negatively associated with other bacteria ${ }^{28}$.

Due to the nutritional demand and oxygen tension, bacteria present in coronal segment differ from that present in apical segment of the same infected tooth. Genius Lactobacillus in the apical samples and genus Actinomyces in the coronal samples are most abundantly present. Anaerovorax was significantly more abundant in the coronal samples than in the apical samples. Rocas et al. 2010 found Streptococci more often in the coronal part and Prevotella baroniae, Tannerella forsythia, and Fusobacterium nucleatum more often in the apical segment of the $\operatorname{root}^{29}$. Endodontic microorganisms also differ according to the clinical presentation of the lesion. See table 2 In symptomatic teeth, most common phyla present are Actinobacteria and Proteobacteria and most common species were Propionibacterium acidifaciens, Propionibacterium propionicum, Streptococcus sanguinis, Propionibacterium acnes, Neisseria macacae. In asymptomatic teeth, dominant phyla are Bacteroides, Firmicutes, Fusobacteria, Spirochetes, and Synergistetes and common species were Pyramidobacter piscolens, Rothia dentocariosa, Tannerella forsythia, Phocaeicola abscessus, Leptotrichia trevisanii Wee Tees $^{30}$. 
Table 2: Bacterial species in endodontic infection presenting with different clinical symptoms.

\begin{tabular}{ll}
\hline Preoperative pain & Fusobacterium nucleatum, gram-negative bacilli, Streptococcus sp. \\
\hline Postoperative pain & gram positive cocci, Streptococcus sp., facultative anaerobes \\
\hline Periapical abscesses & P.gingivalis, P. intermedia, P.nigrescens \\
\hline Presence of pain & A. viscosus, S. sanguis \\
\hline Pain on palpation & Staphylococcus haemolyticus, Veillonella spp. \\
\hline Pain to percussion & Actinomyces spp., A. naeslundii, A. viscosus. \\
\hline Periapical swelling & S. mitis, Bacteroides spp., Veillonella spp. \\
\hline Presence of sinus tract & Neisseria spp., Staphylococcus haemolyticus. \\
\hline Wet canal & P. acnes, Bacteroides spp. \\
\hline Periapical bone resorption & T. denticola \\
\hline Symptomatic cases only & P. intermedia \\
\hline $\begin{array}{l}\text { Asymptomatic chronic } \\
\text { apical periodontitis and } \\
\text { Secondary endodontic } \\
\text { infection in failing cases }\end{array}$ & E. faecalis \\
\hline
\end{tabular}

\section{Recurrent Infection}

Most endodontic treatment failures occur due to incomplete eradication of infection from the root canal and unsatisfactory standard control of infection. Anatomical diversity and improper technique result in incomplete eradication of infection from root canal during treatment. Areas in the root canal like isthmus ramifications, deltas, canal irregularities, lateral canal and dentinal tubules which are clinically difficult for instrumentation and disinfection provides residual organic and inorganic matter in the canal.

These residual organic and inorganic matters provide a suitable substrate for residual bacteria in those areas (known as persistent intraradicular infection). When complete eradication of these residuals substrate is performed, still the failure of endodontic treatment is found. This is due to the incomplete coronal and apical seal from where fluid infiltrate in the canal providing the suitable substrate for bacterial growth (known as secondary intraradicular infection). Failure in chemomechanical preparation, failure to maintain proper sterilization, incomplete coronal and apical seal, limit and quality of root filling materials favor the survival of microorganisms after the treatment or re-infection of the canal leading to endodontic treatment failure ${ }^{31}$.

Mechanical and chemical injuries are often associated with an iatrogenic factor for recurrent infection. The most common cause of interappointment flare-ups is due to the extrusion of microorganisms and their products to the periapical region during the instrumentation and irrigation of the root canal.

Changes in endodontic microbiota and/or in environmental conditions and an increase of the oxidation-reduction potential due to microorganisms also induce flare-ups ${ }^{32}$. The complete aseptic condition must be maintained during the procedure to prevent secondary infection. The study found the presence of $\mathrm{P}$. acnes and coagulase-negative Staphylococci, including S. epidermidis, in endodontic failure cases which has been found in the gloves used during endodontic procedure $^{33}$.

The primary endodontic infection is polymicrobial. It consists mainly of gram-negative bacteria with a small proportion of gram-positive bacteria (see Table 3) that are resistant to root canal treatment and his the ability to adapt the harsh environmental 
condition in endodontically treated canals ${ }^{34}$. Poorly treated root canal contains the organic and inorganic residuals and the microflora which on suitable environment become more viable. These incompletely treated root canals harbor the similar microflora as in primary infection that may contain up to 30 species. But in the properly treated root canal, it shows monomicrobial infection usually contain restricted $1-5$ species ${ }^{35}$.

The strains isolated from recurrent infection consisted of facultative anaerobic and obligate anaerobic bacterial species. Helicobacter pylori were also detected in persistent endodontic infection as the most prevalent species $^{36}$. Gram-positive
Facultative anaerobes, especially Enterococcuss spp, are the most frequently isolated species but in some research number of this species was very few or even not detected. Species belonging to genera Actinomyces, Propionibacterium propionicum, and Enterococcus faecalis are the most frequently isolated microbes in the reinfection of previously treated root canal ${ }^{35}$.

A monoinfection of $\mathrm{E}$. faecalis was found after intracanal dressing with calcium hydroxide, and a monoinfection of $\mathrm{A}$. viscosus was found after intracanal dressing with Ledermix ${ }^{37}$. One of the studies has detected Actinomyces radicidentis associated with failure of root canal treatment ${ }^{38}$.

\section{Table 3: Bacteria present in recurrent endodontic infection}

\section{Gram positive bacteria}

Enterococcus faecalis

Actinomyces spp

Propionibacterium propionicum

Streptococcus spp (S. mitis, S. anginosus, S. oralis., S. gordonii)

Staphylococcus spp

Lactobacillus (L. paracasei, L. acidophilus)

Olsenella uli

Parvimonas micra

Pseudoramibacter alactolyticus

Bifidobacterium spp

Eubacterium spp

\section{Gram-negative anaerobic bacteria}

Fusobacterium nucleatum

Prevotella spp

Campylobacter rectus

Dialister spp (D.pneumosintes, D. invisus)

Tanerella forsythia 
The phyla found in highest levels were Firmicutes, Proteobacteria, and Bacteroidetes. The bacteria found in these cases are predominantly Grampositive coccus, rods, and filaments. The most commonly isolated gram-positive cocci include the Streptococcus spp, E. faecalis, and Peptostreptococcus spp. The most frequently detected facultative anaerobic cocci are Enterococcus faecalis, Streptococcus spp., Staphylococcus spp. Facultative anaerobic rods are Lactobacillus spp., Actinomyces spp., Enterobacter spp., Pseudomonas spp. , Actinobacter baumanii ${ }^{35}$, 39,40 In one of the study, from 32 dental samples only 4 Enterococcus faecalis were isolated along with viridans streptococci together with E. faecalis strain and gram-negative rods and Neisseria sp. as well ${ }^{41}$. Twenty-eight phylotypes were detected in more than one sample, revealing a high inter-sample variability. Parvimonas micra, Solobacterium moore, Dialister invisus, Enterococcus faecalis, Filifactor alocis, and Fusobacterium nucleatum were the prevalent species ${ }^{40}$.

Variation in clinical symptoms varies with the microorganism such as Moraxella osloensis in mild sensation of pain, A. rimae, A. prevotii, P. alactolyticus, D. invisus, and F.nucleatum in chronic apical abscess ${ }^{37}$. Microorganisms present in secondary infection is different from that in primary infection with same clinical presentation (Table 4).

Table 4: Bacterial species significantly associated with signs and symptoms in primary infection and recurrent endodontic infection.

\begin{tabular}{lll}
$\begin{array}{l}\text { Signs and } \\
\text { symptoms }\end{array}$ & $\begin{array}{l}\text { Bacterial species } \\
\text { (primary infection) }\end{array}$ & $\begin{array}{l}\text { Bacterial species } \\
\text { (recurrent infection) }\end{array}$ \\
\hline Pain & Actinomyces viscosus & Porphyromonas spp \\
& Streptococcus sanguis & Prevotella spp \\
& & Peptostreptococcus magna \\
& & Peptostreptococcus micros
\end{tabular}

$\begin{array}{lll}\text { Tender to } & \text { Actinomyces spp } & \text { Peptostreptococcus spp } \\ \text { percussion } & \text { Actinomyces naeslundii } & \text { Eubacterium spp } \\ & \text { Actinomyces viscosus } & \text { Porphyromonas gingivalis } \\ & & \text { Prevotella spp }\end{array}$

\begin{tabular}{lll}
\hline Abscess & A Actinomyces spp & Treponema denticola \\
Actinomyces naeslundii & Tannerella forsythiasis \\
Bacteroides spp & Dialister peumosintes \\
Corynebacterium spp & \\
Propionibacterium acnes &
\end{tabular}

Interappointment flare-up is more in necrotic tissue than vital pulp. Treated canal, when left open will have easy access to the microorganisms from the oral cavity. Presence of planctomycete and 
nitrospira in Koreans ${ }^{30}$ and Moraxella osloensis only in German population can be linked with open treated canal where these microbes get an entry from their food ${ }^{33}$. Enterococcus faecalis is the most commonly isolated bacteria around $70 \%$ of the failed endodontically treated canal. E. faecalis may be present in the canal as a primary infection and/or on suitable condition may invade the canal during the treatment procedure iatrogenically or via food taken by the patient during the process of treatment ${ }^{40}$. E. faecalis is exogenous in origin commonly found in the milk products or in certain fermented food products such as sausages and olives $^{39,41}$. However, the experiment has found a different strain of E. faecalies in the root canal and in milk products ${ }^{42}$.

Most commonly isolated yeast is of Candida species. Candida albicans is the most frequently detected yeasts in persistent endodontic infection .C. albicans, C. glabrata, C. guilliermondii, C. inconspicua and Geotrichum candidum are other Candida species detected from root canals ${ }^{43}$. It is rare inhabitants which progress in the canal when the canal is exposed to the oral cavity. The candidal organisms may be lodged in the dentinal tubules and overgrow in the favorable condition or may have entered the canal during primary treatment or posttreatment due to inadequate coronal $\mathrm{seal}^{44}$. C. albicans and E.faecalis survive as mono-infection, in the nutritionally deprived environment and also can withstand the antimicrobial action of calcium hydroxide ${ }^{43}$.

Actinomyces species comprised $15 \%$ of the microflora and the dominant species were A. israelii. A.meyerii and A. radicidentis. Propionibacterium propionicum, its pathogenic potential may be similar to that exhibited by Actinomyces $^{45}$. It may be due to its virulence that progresses it for extraradicular infection. Periapical actinomycosis and presence of Propionibacterium propionicum cannot be treated by conventional root canal treatment and require periapical surgery. $T$. denticola, T. socranskii subspecies socranskii, T. maltophilum, T. lecithinolyticum was detected spirochetes $^{46}$.

\section{Conclusion}

Dental diseases are mainly associated with the virulence of different microorganisms present in the oral cavity. After the initiation of disease, exogenous bacteria are attracted to the site. Thus maintenance of proper oral hygiene prevents the transformation of normal flora to a diseased state. If the disease is diagnosed early and treated, it prevents from the life-threatening condition. These variations of prevalent species may be due to sensitivity and specificity of identification method, sampling technique, geographic location, and personal habits.

Nosocomial infection has found to be associated with persistent endodontic infection. Thus, proper sterilization and change of instruments and gloves after each procedure for every root canal instrumentation are mandatory to provide clean sterile preparation of the canal. Persistent/secondary intraradicular infection associated with Actinomyces species and P. propionicum, is treated with surgical procedure only. Thus bacteriological analysis of the canal helps to determine the efficacy of the endodontic treatment in primary infection, chances of secondary or persistent endodontic infection, and requirement of surgical treatment. Thus the knowledge of the microbiology of the disease provides the proper treatment plan.

\section{Conflicts of interest}

None.

\section{Acknowledgment}

None.

\section{Funding}

None

\section{References}

1. Parahitiyawa NB, Scully C, Leung WK, Yam WC, Jin LJ, Samaranayake LP. Exploring the oral bacterial flora: current status and future directions. Oral Diseases. 2010;16(2):136-45. 
2. Xu X, He J, Xue J, Wang Y, Li K, Zhang K, et al. Oral cavity contains distinct niches with dynamic microbial communities. Environmental Microbiology. 2015;17(3):699-710.

3. Kilian M, Chapple IL, Hannig M, Marsh PD, Meuric $\mathrm{V}$, Pedersen AM, et al. The oral microbiome - an update for oral healthcare professionals. British dental journal. 2016;221(10):657-66.

4. Aas JA, Paster BJ, Stokes LN, Olsen I, Dewhirst FE. Defining the normal bacterial flora of the oral cavity. Journal of clinical microbiology. 2005;43(11):572132.

5. Schulze-Schweifing K, Banerjee A, Wade WG. Comparison of bacterial culture and 16S rRNA community profiling by clonal analysis and pyrosequencing for the characterization of the dentine caries-associated microbiome. Frontiers in cellular and infection microbiology. 2014;4:164.

6. Wolff D, Frese C, Maier-Kraus T, Krueger T, Wolff B. Bacterial biofilm composition in caries and caries-free subjects. Caries research. 2013;47(1):69-77.

7. Belstrom D, Fiehn NE, Nielsen CH, Holmstrup P, Kirkby N, Klepac-Ceraj V, et al. Altered bacterial profiles in saliva from adults with caries lesions: a casecohort study. Caries research. 2014;48(5):368-75.

8. Henne K, Rheinberg A, Melzer-Krick B, Conrads G. Aciduric microbial taxa including Scardovia wiggsiae and Bifidobacterium spp. in caries and caries free subjects. Anaerobe. 2015;35(Pt A):60-5.

9. Koo H, Bowen WH. Candida albicans and Streptococcus mutans: a potential synergistic alliance to cause virulent tooth decay in children. Future microbiology. 2014;9(12):1295-7.

10. Kianoush N, Adler CJ, Nguyen KA, Browne GV, Simonian M, Hunter N. Bacterial profile of dentine caries and the impact of $\mathrm{pH}$ on bacterial population diversity. PloS one. 2014;9(3):e92940.

11. Zhou J, Jiang N, Wang S, Hu X, Jiao K, He X, et al. Exploration of Human Salivary Microbiomes--Insights into the Novel Characteristics of Microbial Community Structure in Caries and Caries-Free Subjects. PloS one. 2016;11(1):e0147039.

12. Ma C, Chen F, Zhang Y, Sun X, Tong P, Si Y, et al. Comparison of oral microbial profiles between children with severe early childhood caries and caries-free children using the human oral microbe identification microarray. PloS one. 2015;10(3):e0122075.

13. Zaremba ML, Stokowska W, Klimiuk A, Daniluk T, Rozkiewicz D, Cylwik-Rokicka D, et al. Microorganisms in root carious lesions in adults.
Advances in medical sciences. 2006;51 Suppl 1:23740.

14. Lif Holgerson P, Ohman C, Ronnlund A, Johansson I. Maturation of Oral Microbiota in Children with or without Dental Caries. PloS one. 2015;10(5):e0128534.

15. Tanner AC, Kent RL, Jr., Holgerson PL, Hughes CV, Loo CY, Kanasi E, et al. Microbiota of severe early childhood caries before and after therapy. Journal of dental research. 2011;90(11):1298-305.

16. Hsiao WW, Li KL, Liu Z, Jones C, Fraser-Liggett CM, Fouad AF. Microbial transformation from normal oral microbiota to acute endodontic infections. BMC genomics. 2012;13:345.

17. Triches TC, de Figueiredo LC, Feres M, de Freitas SF, Zimmermann GS, Cordeiro MM. Microbial profile of root canals of primary teeth with pulp necrosis and periradicular lesion. Journal of dentistry for children (Chicago, Ill). 2014;81(1):14-9.

18. Siqueira JF, Jr., Rocas IN. Distinctive features of the microbiota associated with different forms of apical periodontitis. Journal of oral microbiology. 2009;1.

19. Galhotra V, Dey S, Priyank H, Paranjape T, Sharma N, Singh I. Prevalence of Microorganisms in Root Canals of Human Permanent Teeth with Symptomatic Nonvital Pulp and Chronic Periapical Lesions: A Microbiological Study. Journal of International Oral Health. 2015;7(11):71.

20. Al-Samahi S, Al-Omari MA. Detection of bacteria in endodontic samples and its association with defined clinical signs and symptoms of endodontic infection. Saudi Journal of Oral Sciences. 2014;1(2):83.

21. Santos AL, Siqueira JF, Jr., Rocas IN, Jesus EC, Rosado AS, Tiedje JM. Comparing the bacterial diversity of acute and chronic dental root canal infections. PloS one. 2011;6(11):e28088.

22. Shang JJ, Yang QB, Zhao HY, Cai S, Zhou Y, Sun Z. Preliminary molecular analysis of bacterial composition in periapical lesions with primary endodontic infections of deciduous teeth. Chinese medical journal. 2013;126(16):3112-7.

23. Siqueira JF. Periapical actinomycosis and infection with Propionibacterium propionicum. Endodontic Topics. 2003;6(1):78-95.

24. Fabricious L, Dahlen G, Öhman AE, Moller AJ. Predominant indigenous oral bacteria isolated from infected root canals after varied times of closure. European Journal of Oral Sciences. 1982;90(2):134-44.

25. Sundqvist G. Taxonomy, ecology, and pathogenicity of the root canal flora. Oral surgery, oral medicine, and oral pathology. 1994;78(4):522-30. 
26. Marsh PD. Are dental diseases examples of ecological catastrophes? Microbiology (Reading, England). 2003;149(Pt 2):279-94.

27. Sundqvist G. Associations between microbial species in dental root canal infections. Oral microbiology and immunology. 1992;7(5):257-62.

28. Sundqvist G, Figdor D. Life as an endodontic pathogen. Endodontic Topics. 2003;6(1):3-28.

29. Ozok AR, Persoon IF, Huse SM, Keijser BJ, Wesselink PR, Crielaard W, et al. Ecology of the microbiome of the infected root canal system: a comparison between apical and coronal root segments. International endodontic journal. 2012;45(6):530-41.

30. Lim S-M, Lee T-K, Kim E-J, Park J-H, Lee Y, Bae K$\mathrm{S}$, et al. Microbial profile of asymptomatic and symptomatic teeth with primary endodontic infections by pyrosequencing. Journal of Korean Academy of Conservative Dentistry. 2011;36(6):498-505.

31. Nobrega LM, Delboni MG, Martinho FC, Zaia AA, Ferraz CC, Gomes BP. Treponema diversity in root canals with endodontic failure. European journal of dentistry. 2013;7(1):61-8.

32. Siqueira JF, Jr. Microbial causes of endodontic flareups. International endodontic journal. 2003;36(7):45363.

33. Tennert C, Fuhrmann M, Wittmer A, Karygianni L, Altenburger MJ, Pelz K, et al. New bacterial composition in primary and persistent/secondary endodontic infections with respect to clinical and radiographic findings. Journal of endodontics. 2014;40(5):670-7.

34. Murad CF, Sassone LM, Faveri M, Hirata R, Jr., Figueiredo L, Feres M. Microbial diversity in persistent root canal infections investigated by checkerboard DNA-DNA hybridization. Journal of endodontics. 2014;40(7):899-906.

35. Dudeja PG, Dudeja KK, Srivastava D, Grover S. Microorganisms in periradicular tissues: Do they exist? A perennial controversy. Journal of oral and maxillofacial pathology : JOMFP. 2015;19(3):356-63.

36. Niazi SA, Vincer L, Mannocci F. Glove Contamination during Endodontic Treatment Is One of the Sources of Nosocomial Endodontic Propionibacterium acnes
Infections. Journal of endodontics. 2016;42(8):120211.

37. Narayanan LL, Vaishnavi C. Endodontic microbiology. Journal of conservative dentistry : JCD. 2010;13(4):233-9.

38. Kalfas S, Figdor D, Sundqvist G. A new bacterial species associated with failed endodontic treatment: identification and description of Actinomyces radicidentis. Oral surgery, oral medicine, oral pathology, oral radiology, and endodontics. 2001;92(2):208-14.

39. Vidana R, Sullivan A, Billstrom H, Ahlquist M, Lund B. Enterococcus faecalis infection in root canals - hostderived or exogenous source? Letters in applied microbiology. 2011;52(2):109-15.

40. Zhang C, Hou BX, Zhao HY, Sun Z. Microbial diversity in failed endodontic root-filled teeth. Chinese medical journal. 2012;125(6):1163-8.

41. Kovac J, Kovac D, Slobodnikova L, Kotulova D. Enterococcus faecalis and Candida albicans in the dental root canal and periapical infections. Bratislavske lekarske listy. 2013;114(12):716-20.

42. Vidana R, Rashid MU, Ozenci V, Weintraub A, Lund B. The origin of endodontic Enterococcus faecalis explored by comparison of virulence factor patterns and antibiotic resistance to that of isolates from stool samples, blood cultures and food. International endodontic journal. 2016;49(4):343-51.

43. Waltimo T, Sen B, Meurman JH, Ørstavik D, Haapasalo M. Yeasts in apical periodontitis. Critical Reviews in Oral Biology \& Medicine. 2003;14(2):12837.

44. Kumar J, Sharma R, Sharma M, Prabhavathi V, Paul J, Chowdary CD. Presence of Candida albicans in Root Canals of Teeth with Apical Periodontitis and Evaluation of their Possible Role in Failure of Endodontic Treatment. Journal of international oral health : JIOH. 2015;7(2):42-5.

45. Yeung M. Molecular and genetic analyses of Actinomyces spp. Critical Reviews in Oral Biology \& Medicine. 1999;10(2):120-38.

46. Sakamoto M, Siqueira JF, Jr., Rocas IN, Benno Y. Diversity of spirochetes in endodontic infections. Journal of clinical microbiology. 2009;47(5):1352-7. 